Relations industrielles

Industrial Relations

\title{
Administrative Action, by William H. Newman. N.Y., Prentice-Hall, 1950, pp. 460.
}

\section{Louis-Marie Tremblay}

Volume 13, numéro 1, janvier 1958

URI : https://id.erudit.org/iderudit/1022482ar

DOI : https://doi.org/10.7202/1022482ar

Aller au sommaire du numéro

Éditeur(s)

Département des relations industrielles de l’Université Laval

ISSN

0034-379X (imprimé)

1703-8138 (numérique)

Découvrir la revue

Citer ce compte rendu

Tremblay, L.-M. (1958). Compte rendu de [Administrative Action, by William H. Newman. N.Y., Prentice-Hall, 1950, pp. 460.] Relations industrielles / Industrial Relations, 13(1), 114-114. https://doi.org/10.7202/1022482ar

Tous droits réservés @ C Département des relations industrielles de l’Université Laval, 1958
Ce document est protégé par la loi sur le droit d'auteur. L’utilisation des services d'Érudit (y compris la reproduction) est assujettie à sa politique d'utilisation que vous pouvez consulter en ligne.

https://apropos.erudit.org/fr/usagers/politique-dutilisation/ 
Le Code innove également en matière de formation professionnelle, d'orientation professionnelle et d'apprentissage.

Enfin, le Code du Travail établit une Régie des prix dont la juridiction s'étend aux producteurs primaires (à l'exception des cultivateurs), aux fabricants et aux grossistes. Il ne s'agit pas d'un organisme de contrôle des prix, ni d'une système d'arbitrage obligatoire des prix, mais d'un organisme permanent d'enquête dont le but est d'éclairer l'opinion publique en matière de prix.

Le Code du Travail est en vente au bureau de M. Gérard Picand, 8227, boulevard St-Laurent, Montréal.

Administrative Action, by William $\mathbf{H}$. Newman. N.Y., Prentice-Hall, 1950, pp. 460.

Tel que suggéré par l'entête, ce volume traite des principes et techniques d'une administration dynamique. Il s'adresse spécialement aux administrateurs des grandes corporations modernes sans toutefois exclure ceux des entreprises plus modestes, en leur fournissant les moyens conceptuels et pratiques de s'adapter aux changements techniques et sociaux afin de préserver et promouvoir les valeurs propres à notre civilisation occidentale contemporaine. "Administrative Action 》 s'avèrera très utile au personnel de direction, car il contient une analyse pénétrante et compréhensive des différentes fonctions administratives, et apporte de nouvelles idées pour améliorer les procédures de direction.

Le livre se divise en cinq parties, dont chacune discute l'un des éléments de base de toute administration: la planification, l'organisation, l'assemblement des ressources, la direction et le contrôle.

L'administration d'une entreprise exige avant tout de planifier les opérations, budgets, standards, etc. Le responsable sera plus efficace s'il connaît bien les diverses formes de planification, et s'il peut choisir la meilleure se- lon les caractéristiques des phénomènes et les conditions ambiantes. Dans les chapitres deux à cinq, la nature, les avantages et les désavantages des types de planification utilisés sont décrits. Les deux chapitres suivants exposent comment prendre les décisions dont dépendent les succès ou la faillite des programmes, même les mieux conçus.

La deuxième partie sur l'organisation est assurément la meilleure de l'ouvrage. L'auteur lui consacre d'ailleurs la plus large part de son espace. D'un côté, les éléments de toute organisation y sont individuellement analysés de façon claire et détaillée. Newman couvre à tour de rôle la division départementale, la division des services, la délégation d'autorité, le rôle des spécialistes (staff), les comités, la décentralisation, les bureaux de direction et la représentation des intérêts. A notre avis, les chapitres sur la délégation de l'autorité et le «staff» méritent une mention particulière, et beaucoup d'administrateurs auraient profit à les lire et suivre leurs enseignements. Cette partie se termine par une synthèse de l'organisation structurelle qui permet de considérer les éléments de l'organisation comme un tout intégré.

Les deux parties qui suivent sont plus faibles et moins élaborées que les précédentes. L'une traite de la détermination des besoins de personnel, du choix, de l'évaluation, de la formatiors et de la rémunération des administrateurs. L'autre analyse brièvement la direction et la coordination à travers la supervision. La dernière partie porte sur les étapes du contrôle, avec un chapitre spécial sur le contrôle budgétaire, l'un des instruments les plus efficaces de la direction, et se termine par une analyse de la motivation qui exprime le besoin d'une meilleure compréhension de la motivation humaine dans le processus administratif.

«Administrative Action》 est un bon livre qui vaut mieux que les manuels habituellement servis. Il ne déparera pas les rayons de celui qui s'intéresse aux questions administratives.

L.-M. Tremblay 